\title{
Curvas de Resposta e Isobolograma como Forma de Descrever a ASSOCIAÇÃO DE HERBICIDAS INIBIDORES DO FOTOSSISTEMA II E DA Síntese de Carotenóides ${ }^{1}$
}

\author{
Dose-Response Curve and Isobologram Used to Describe the Mixture of Herbicides Inhibitors of \\ the Photosystem II and Carotenoid Synthesis
}

KRUSE, N.D. ${ }^{2}$, VIDAL, R.A. ${ }^{3}$ e TREZZI, M.M. ${ }^{4}$

\begin{abstract}
RESUMO - A metodologia de curvas de resposta e isobologramas, feitos a partir dos $\mathrm{I}_{50}$ gerados pelas curvas, tem sido bastante apregoada na comprovação de sinergismo. O experimento foi conduzido em casa de vegetação, com girassol cultivado em solução nutritiva (Hoagland), aplicando-se na solução 27 tratamentos, com os herbicidas metribuzin nas doses de 0, 70, 140, 280, 560 e 1.130 nM e clomazone nas doses de 0, 40, 80, 160, 320 e 640 mM. Combinaram-se as doses dos herbicidas de maneira a produzir os $\mathrm{I}_{50}$ das seis curvas resultantes das associações. Nas plantas coletadas, cinco dias após a aplicação, determinou-se o percentual de dano, a massa fresca e a massa seca das plantas de girassol. Os seis pontos de $I_{50}$ de cada variável foram utilizados para formar isobologramas, onde os $I_{50}$ dos herbicidas aplicados isolados, unidos por uma reta, formaram a isobole de aditividade. Em torno desta, foram traçadas faixas de confiança, a partir do produto dos intervalos de confiança dos $I_{50}$ dos herbicidas isolados, dentro das quais se posicionaram os $\mathrm{I}_{50}$ resultantes das associações. Todas as associações situaram-se na região de sinergismo, abaixo da isobole de aditividade, embora com diferentes probabilidades. Na variável massa seca, a associação das doses de clomazone com $140 \mathrm{nM}$ de metribuzin demonstrou probabilidade de, em torno de 90\%, ser uma interação sinérgica.
\end{abstract}

Palavras-chave: mistura de herbicidas, sinergismo, metribuzin, clomazone.

\begin{abstract}
The Dose-Response Curve and Isobolograms methodology, based on the $I_{50}$ generated by curves has been largely recommended to demonstrate synergism. The experiment was conducted in a greenhouse, with sunflower cultivated in nutrient solution (Hoagland), applying in solution 27 treatments, with the herbicides metribuzin, at the rates of $0,70,140,280,560$ and 1,130 nM and clomazone, at the rates of $0,40,80,160,320$ and $640 \mathrm{mM}$. The herbicide doses were combined so as to produce the $I_{50}$ of six resulting curves from the matches. Injury percentage, and fresh and dry weight of sunflower plants were determined five days after application on the collected plants. The six points of $I_{50}$ from each variable were used to obtain isobolograms, where the $I_{50}$ of herbicides applied alone, jointed by a straight line, formed the isobole of additivity. Around of it, confidences strips were drawn from the product of confidences intervals of isolated herbicides $I_{50}$ 's, where were positioned the $I_{50}$ resulting from the mixtures. All the $I_{50}$ mixtures were positioned in the synergism zone, below the isobole of additivity, although with different probabilities. For dry mass variable, the mixture of clomazone rates with $140 \mathrm{nM}$ of metribuzin showed probability around 90\% of being a synergistic interaction.
\end{abstract}

Keywords: herbicide mixture, synergism, metribuzin, clomazone.

Recebido para publicação em 7.10.2005 e na forma revisada em 4.8.2006.

2 Prof. do Dep. de Defesa Fitossanitária, Universidade Federal de Santa Maria - UFSM, 97110-900 Santa Maria-RS, <nkruse@terra.com.br>. ${ }^{3}$ Prof. do Dep. de Plantas de Lavoura, Universidade Federal do Rio Grande do Sul - UFRGS, <ribas.vidal@ufrgs.br>. ${ }^{4}$ Prof. da Universidade Federal Tecnológica do Paraná - UFTPR, 85503-390 Pato Branco-PR, $<$ mtrezzi@brturbo.com.br>. 


\section{INTRODUÇÃO}

A associação de dois ou mais herbicidas para o controle de plantas daninhas em uma mesma cultura é prática bastante usual nas últimas décadas. O tipo de interação decorrente da associação é matéria de constante preocupação e investigação. Apesar de várias nomenclaturas adotadas para classificar esses tipos de interação, os termos aditividade, sinergismo e antagonismo são os mais aceitos. Várias metodologias foram propostas e empregadas para a definição de qual tipo de interação ocorre em uma determinada associação. Na última década, tem sobressaído a proposta do uso de curvas de resposta às doses dos herbicidas e de isobologramas para a definição do tipo de interação (Gowing, 1960; Hatzios \& Penner, 1985; Green \& Bailey, 1987; Gressel, 1990; Streibig et al., 1998, 1999).

A curva de resposta às doses é bastante empregada não só no estudo de associações de herbicidas, mas também na comprovação de resistência de plantas a herbicidas e na influência de fatores ambientais no seu desempenho. Consiste em descrever a resposta biológica de uma planta daninha às doses crescentes de um herbicida isolado e em associação, seguindo um fator constante de diluição, de forma que se obtenham doses eqüidistantes em escala logarítmica. Normalmente, obtém-se uma curva simétrica em formato sigmoidal, que pode ser ajustada pelo modelo logístico. Desse ajuste obtém-se a estimativa da dose que provoca $50 \%$ do efeito total possível na variável resposta analisada (Chism et al., 1992; Green \& Streibig, 1993; Seefeldt et al., 1995; Blackshaw et al., 1996; Johnson \& Young, 2002).

A representação gráfica das doses isoladas e em associação que causam 50\% (ou outro percentual) de efeito $\left(I_{50}\right)$ de dois agentes químicos é chamada de isobolograma. A descrição da ação conjunta de dois produtos por esse método permite que os princípios e problemas ligados à análise de associações possam ser claramente percebidos.

O isobolograma foi inicialmente empregado na farmacologia e trazido para a área de defensivos agrícolas por Tammes (1964), na qual, especialmente no estudo de sinergismo, essa representação pode ser de grande utilidade. No isobolograma, os $I_{50}$ oriundos da aplicação isolada de cada produto são unidos, gerando a isobole de aditividade ou linha teórica de aditividade. Os demais $\mathrm{I}_{50}$ obtidos da associação em diferentes proporções dos produtos podem então ser analisados em relação à sua posição diante da isobole de aditividade. Se esses pontos se posicionarem em torno da isobole de aditividade, a ação é de aditividade; ao se colocarem abaixo, a ação é de sinergismo; ao se situarem acima, a ação é de antagonismo. A posição relativa desses pontos pode ser mais bem avaliada com a representação dos intervalos de confiança entre os $\mathrm{I}_{50}$ obtidos. Assim, em torno da isobole de aditividade forma-se uma faixa de confiança e, em torno $\operatorname{dos} I_{50}$ das associações, intervalos de confiança, exprimindo a amplitude de variação da dose estimada (Tammes, 1964; Morse, 1978; Drury, 1980; Campbell et al., 1981; Green \& Bailey, 1987; Streibig, 1987; Green \& Streibig, 1993; Gessner, 1995; Streibig et al., 1998).

Uma forma complementar de análise das doses $I_{50}$ para os produtos aplicados isolados e em associação é a do cálculo do índice de combinação $\left(\mathrm{I}_{\text {comb }}\right)$. Este índice segue o princípio de aditividade de Loewe, relacionando as doses $I_{50}$ empregadas na associação com as doses $I_{50}$ dos produtos isolados. $\mathrm{O}$ índice equivalente à unidade indica aditividade e, menor ou maior, indica sinergismo ou antagonismo, respectivamente (Ramakrishnan \& Jusko, 2001).

As metodologias aqui descritas podem ser de grande utilidade no esclarecimento da interação resultante da associação de herbicidas. Entre as diversas conseqüências advindas da associação, sem dúvida a existência de sinergismo é vantajosa, porque se combina o espectro de ação dos herbicidas, previne-se o surgimento de resistência e aumenta-se a eficiência de controle, permitindo o uso de doses menores (Hatzios \& Penner, 1985; Green, 1989; Gressel, 1990; Wrubel \& Gressel, 1994). Nesse sentido, foram procurados, a partir do conhecimento dos vários mecanismos de ação dos herbicidas, dois mecanismos que pudessem apresentar sinergismo quando associados. Levanta-se a hipótese que, pela natureza de suas ações, herbicidas inibidores do fluxo de elétrons do fotossistema II (FS II) e herbicidas inibidores da síntese de carotenóides apresentem potencial de sinergismo. Enquanto os primeiros são geradores de estresse oxidativo, 
os segundos reduzem a capacidade da planta de combater este estresse. Assim, espera-se que a associação de herbicidas pertencentes a estes dois mecanismos de ação apresente interação sinérgica como resultado final.

A fim de verificar a existência de sinergismo na associação de herbicidas inibidores do fluxo de elétrons no FS II e inibidores da síntese de carotenóides, conduziu-se o presente trabalho com a associação de metribuzin e clomazone, analisando-se a interação resultante com os métodos anteriormente descritos.

\section{MATERIAL E MÉTODOS}

Foi conduzido um experimento em ambiente protegido (estufa plástica), durante o mês de dezembro, com a finalidade de obter as curvas de resposta às doses de metribuzin e clomazone e os isobologramas correspondentes. Para isso, sementes de girassol do híbrido DKB 11 foram colocadas para germinar e, uma semana após, transplantaram-se três plântulas por vaso, com dimensões de $10 \mathrm{~cm}$ de diâmetro, $17 \mathrm{~cm}$ de altura e $1.200 \mathrm{~cm}^{3} \mathrm{de}$ capacidade volumétrica. Os vasos de polietileno tereftalato (PET) foram mantidos revestidos com papel-alumínio. As plântulas foram fixadas em placa de isopor encaixada no vaso. A solução nutritiva utilizada foi preparada de acordo com o proposto por Hoagland \& Arnon, descrito em Hewitt (1966), com as seguintes con centrações: $\mathrm{Ca}\left(\mathrm{NO}_{3}\right)_{2} 4 \mathrm{H}_{2} \mathrm{O}-4 \mathrm{mM}, \mathrm{MgSO}_{4} \mathrm{H}_{2} \mathrm{O}-2 \mathrm{mM}, \mathrm{KNO}_{3}$ - $4 \mathrm{mM},\left(\mathrm{NH}_{4}\right)_{2} \mathrm{SO}_{4}-0,435 \mathrm{mM}, \mathrm{KH}_{2} \mathrm{PO}_{4}-$ $0,5 \mathrm{PM}, \mathrm{MnSO}_{4} \mathrm{H}_{2} \mathrm{O}-2 \mathrm{PM}, \mathrm{CuSO}_{4} 5 \mathrm{H}_{2} \mathrm{O}-$ 0,3 PM, $\mathrm{ZnSO}_{4} 7 \mathrm{H}_{2} \mathrm{O}-0,8 \mathrm{PM}, \mathrm{NaCl}-30 \mathrm{PM}$, $\mathrm{Na}_{2} \mathrm{MoO}_{4} 2 \mathrm{H}_{2} \mathrm{O}-0,1 \mathrm{PM}, \mathrm{H}_{3} \mathrm{BO}_{3}-10 \mathrm{PM}$ e FeEDTA - 10 PM. Foram empregados $1.000 \mathrm{~mL}$ da solução por vaso, mantida com pH 6,5 e sob aeração constante. A cada três dias era completado o volume e verificado o $\mathrm{pH}$, sendo corrigido, quando necessário, com NaOH 1 M.

Os tratamentos herbicidas foram aplicados na solução nutritiva quando as plantas apresentavam o segundo par de folhas completamente expandidas. Preparou-se uma solução herbicida para cada dose testada, de forma que $1 \mathrm{~mL}$ desta solução, diluída no volume do vaso, resultasse na dose desejada. Para aplicação, retirava-se a placa de isopor com as plantas e adicionava-se a solução herbicida, agitando vigorosamente com auxílio de bastão de vidro.
Nos dias subseqüentes à aplicação, as plantas permaneceram em ambiente protegido (estufa plástica), com temperatura máxima média de $36{ }^{\circ} \mathrm{C}$ e mínima média de $20^{\circ} \mathrm{C}$. A radiação teve duração média de 11 h30 (heliógrafo Fuess), com fluxo fotônico fotossintético máximo equivalente a $950 \mathrm{Pmol} \mathrm{m}^{-2} \mathrm{~s}^{-1}$ (sensor quântico acoplado a porômetro Li-Cor 1600).

As combinações necessárias para obtenção das curvas de resposta totalizaram 27 tratamentos, em quatro repetições, com seis doses do herbicida metribuzin e seis doses de clomazone, em delineamento completamente casualizado. Para o metribuzin, empregaramse as doses de 0, 70, 140, 280, 560 e 1.120 nM. Para o clomazone, as doses foram de 0, 40, $80,160,320$ e 640 PM. As doses de 70 e $140 \mathrm{nM}$ de metribuzin foram associadas com todas as doses de clomazone. Da mesma forma, as doses de 40 e 80 PM de clomazone foram associadas com todas as doses de metribuzin. Ao final do quinto dia após a aplicação dos tratamentos, determinou-se o dano nas plantas, valendo-se de escala percentual, em que 0\% representou ausência de dano e 100\%, morte das plantas (Frans \& Crowley, 1986). Realizou-se então a coleta e determinou-se a massa fresca (MF) por vaso e, após secagem em estufa a $60{ }^{\circ} \mathrm{C}$ até massa constante, a massa seca (MS) por vaso.

Os dados obtidos de cada variável foram organizados de maneira a formar três grupos de tratamentos ou curvas de resposta às doses para cada herbicida. Uma curva foi formada pelas seis doses de metribuzin aplicado isoladamente, outra com as mesmas seis doses associadas com 40 PM de clomazone e a terceira com as seis doses associadas com 80 PM de clomazone. Para o clomazone, formou-se uma curva com seis doses do produto isolado, outra com as seis doses em associação com $70 \mathrm{nM}$ de metribuzin e a terceira com as seis doses em associação com 140 nM de metribuzin.

Para análise dos dados de cada grupo de tratamentos foi utilizada a análise da variância e regressão não-linear, ajustando-se o modelo log-logístico (Green \& Streibig, 1993; Seefeldt et al., 1995). Para isso, valeu-se do aplicativo computacional SAS ${ }^{\circledR}$, que emprega em seu "proc nlin" o algoritmo de Gauss-Newton (Motulsky \& Ransnas, 1987; SAS, 1989). Com o ajuste deste modelo obteve-se para cada 
curva o valor $\mathrm{I}_{50}$, em um total de seis $\mathrm{I}_{50}$ para cada variável. Estas doses de $\mathrm{I}_{50}$ foram representadas graficamente, formando um isobolograma com metribuzin versus clomazone (Tammes, 1964; Morse, 1978; Green \& Streibig, 1993; Gessner, 1995).

As doses $\mathrm{I}_{50}$ de cada herbicida aplicado isoladamente, foram unidas, gerando a isobole de aditividade (Morse, 1978; Green \& Streibig, 1993; Gessner, 1995). Em torno da isobole de aditividade foram traçadas linhas unindo os intervalos de confiança (IC) (bilaterais) de 97,5, 95,90 e $80 \%$ de cada um dos $\mathrm{I}_{50}$ de metribuzin e clomazone isolados, sendo denominadas de faixas de confiança (FC). A combinação das probabilidades dos dois intervalos é dada pelo produto destas. A união dos intervalos de confiança de $97,5 \%$ de cada um dos $\mathrm{I}_{50}$ dos produtos isolados, por exemplo, gerou a FC de $95 \%$. Da mesma forma, a faixa de 90\% corresponde à união dos intervalos de 95\%; a de $81 \%$, à união dos intervalos de $90 \%$; e a de $64 \%$, à união dos intervalos de 80\% (Gessner, 1995).

Os quatro $\mathrm{I}_{50}$ resultantes das curvas com associação de metribuzin e clomazone foram representados por círculos cheios e barras verticais ou horizontais, representando seus intervalos de confiança de 95, 90 e $80 \%$. A variável dano sofreu transformação (arcsen $\sqrt{\mathrm{x} \square 0,5}$ ) para corrigir a heterocedasticidade (Riboldi, 1995). Para cada curva ajustada, testou-se a falta de ajuste (Faj.) (Seefeldt et al., 1995), pela expressão:

$$
\text { Faj. }=\left\{\left(S Q_{e r}-S Q_{\text {res. }}\right) /\left(G L_{e r}-G L_{r e s .}\right)\right\} /\left(S Q_{e r .} / G L_{e r .}\right)
$$

sendo $S Q_{\text {er. }}$ a soma de quadrados do erro da análise da variância, $S Q_{r e s}$ a soma de quadrados do resíduo da regressão não-linear, $G L_{e r}$. os graus de liberdade do erro da análise da variância e $G L_{\text {res, }}$ os graus de liberdade do resíduo da regressão não-linear.

Foi também calculado o índice de combinação das associações de herbicidas $\left(\mathrm{IC}_{\text {omb }}\right)$ (Ramakrishnan \& Jusko, 2001), pela expressão:

$$
I C_{\text {omb }}=I_{50 a b 1} / I_{50 a}+I_{50 a b 2} / I_{50 b}
$$

sendo $I_{50 a b 1}$ e $I_{50 a b 2}$ as doses $I_{50}$ dos produtos em associação e $I_{50 a}$ e $I_{50 b}$ as doses $I_{50}$ dos produtos aplicados isoladamente.

\section{RESULTADOS E DISCUSSÃO}

As curvas de resposta da massa fresca (MF) às doses de metribuzin aplicado isoladamente e em associação com 40 e 80 PM de clomazone encontram-se na Figura 1A. Os resultados obtidos nessas três combinações de tratamentos foram adequadamente ajustados ao modelo log-logístico, com teste de falta de ajuste não-significativo. Os três $\mathrm{I}_{50}$ obtidos pelo ajuste do modelo, de 307,7, 183,6 e 108,5 nM,
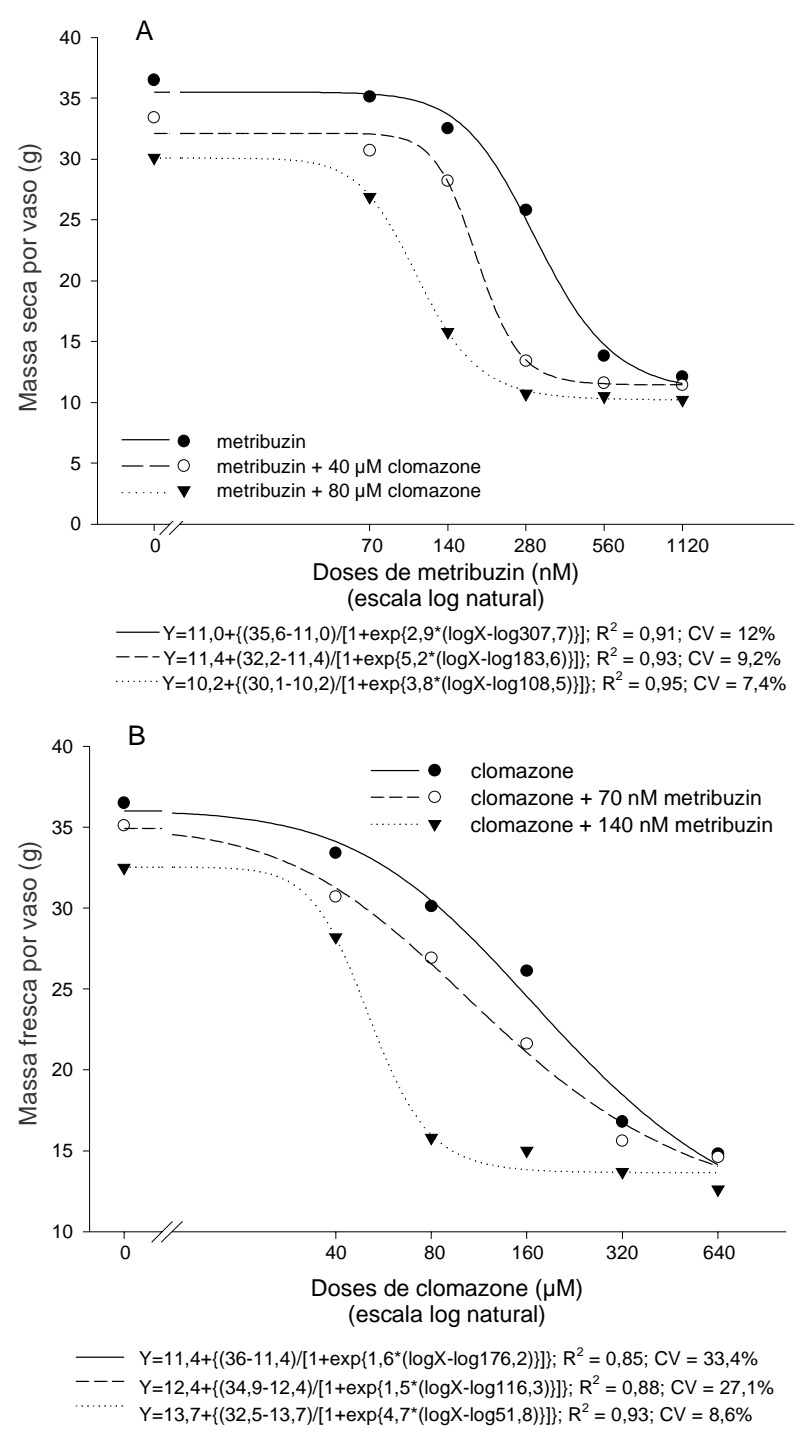

Figura 1 - Curvas de resposta da massa fresca de girassol às doses dos herbicidas metribuzin (A) e clomazone (B), aplicados na solução nutritiva, isolados e em associação, quando as plantas apresentavam o segundo par de folhas completamente expandidas, avaliadas aos cinco dias após a aplicação dos tratamentos. UFRGS, Porto Alegre-RS, 2001. 
respectivamente para metribuzin isolado, metribuzin + 40 PM de clomazone e metribuzin + 80 PM de clomazone, demonstram a contribuição proporcionada pela associação das duas doses de clomazone às seis doses do metribuzin.

Da mesma forma, as curvas de resposta de $\mathrm{MF}$ às doses de clomazone aplicado isolado e em associação com 70 e 140 nM de metribuzin (Figura 1B) também foram bem ajustadas pelo modelo log-logístico. Os $\mathrm{I}_{50}$ obtidos pelo ajuste do modelo aos resultados, de 176,2, 116,3 e 51,8 PM, respectivamente para clomazone isolado, clomazone $+70 \mathrm{nM}$ de metribuzin e clomazone + $140 \mathrm{nM}$ de metribuzin, também refletem a contribuição da associação das duas doses de metribuzin ao desempenho das seis doses de clomazone sobre o girassol.

Na comparação do desempenho dos dois herbicidas, fica evidente a maior capacidade do metribuzin em provocar redução da MF de girassol, uma vez que suas doses estão expressas em unidade mil vezes inferior à unidade de dose do clomazone, embora, proporcionalmente, a redução que o clomazone proporciona nos $\mathrm{I}_{50}$ de metribuzin, da ordem de 40 e $65 \%$, para 40 e 80 PM de clomazone, respectivamente, seja semelhante à redução que metribuzin proporciona nos $I_{50}$ de clomazone, de 34 e $70 \%$, para 70 e $140 \mathrm{nM}$ de metribuzin, respectivamente.

Os coeficientes de determinação das seis curvas com valores superiores a 0,85 demonstram que grande parte da variação da $\mathrm{MF}$ foi explicada pelas doses dos herbicidas (Figura 1). Já os coeficientes de variação são razoáveis para esta variável em experimentos dessa natureza (Souza et al., 2000).

A variável massa seca (MS) refletiu o comportamento apresentado pela MF, com valores de $I_{50}$ semelhantes, bem como no teste de falta de ajuste e nos coeficientes de determinação e de variação (Figura 2). Os $I_{50}$ obtidos nas curvas de resposta da MS às doses do metribuzin, de 333,9, 184,5 e 105,6 nM, respectivamente para metribuzin isolado, associado a 40 e 80 PM de clomazone, demonstram a contribuição das duas doses de clomazone (40 e 80 PM) na redução da MS de girassol em resposta aos tratamentos (Figura 2A). Em comparação com os $\mathrm{I}_{50}$ obtidos com a variável
MF, há equivalência entre os valores, demonstrando haver coerência entre essas duas variáveis.

Na curva de resposta da MS às doses de clomazone também se observou contribuição do metribuzin (70 e $140 \mathrm{nM}$ ) associado às doses de clomazone (Figura 2B). A redução da MS em resposta às doses de clomazone foi acentuada quando associadas com metribuzin. Os valores de $I_{50}$, de 186, 106 e 54,4 PM, foram também bastante próximos aos obtidos
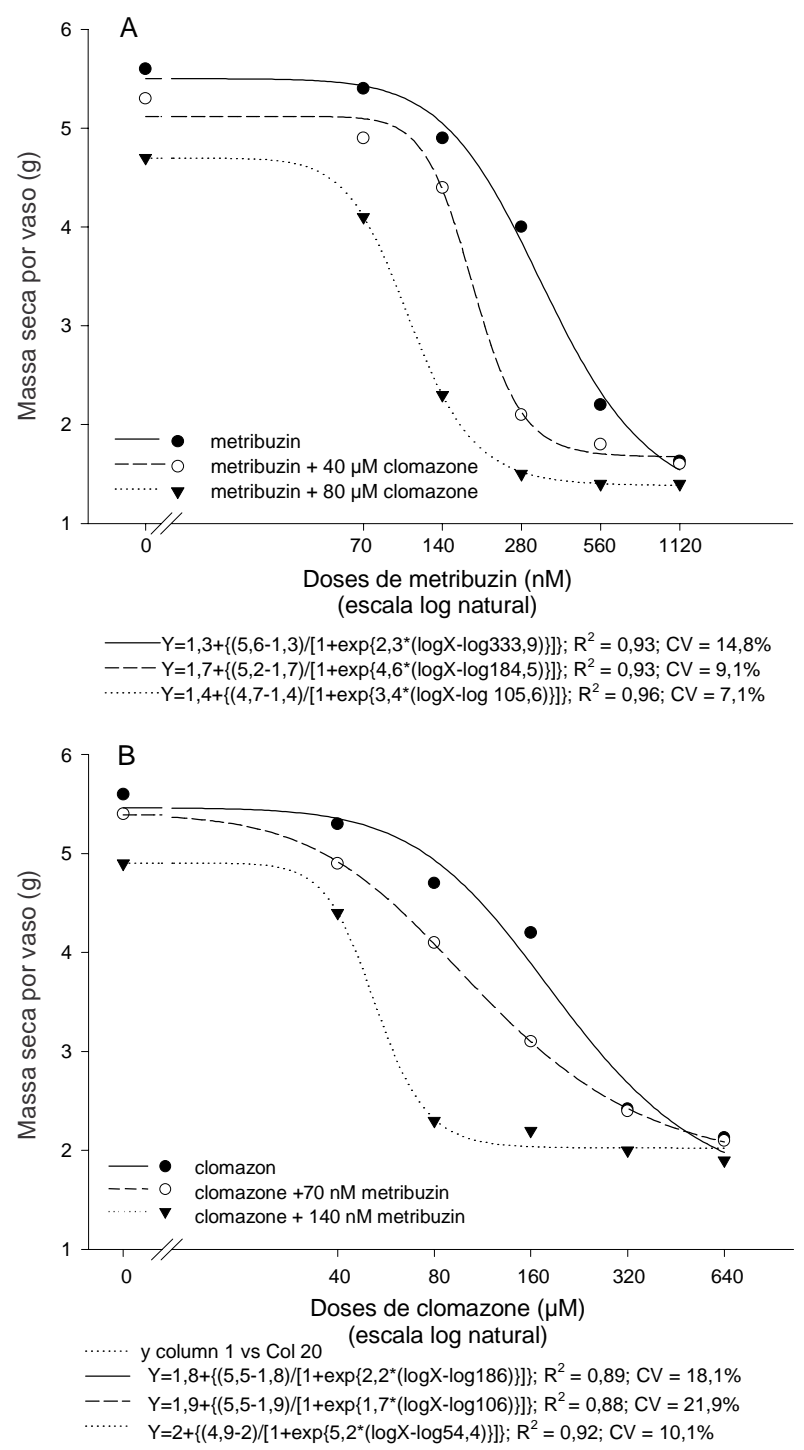

Figura 2 - Curvas de resposta da massa seca de girassol às doses dos herbicidas metribuzin (A) e clomazone (B), aplicados na solução nutritiva, isolados e em associação, quando as plantas apresentavam o segundo par de folhas completamente expandidas e coleta aos cinco dias após a aplicação dos tratamentos. UFRGS, Porto Alegre-RS, 2001. 
para a variável MF. Avaliando em termos percentuais as reduções proporcionadas pelas associações mútuas, houve equivalência entre elas, da mesma forma que foi observado na variável MF. Comparando-se os coeficientes de variação das curvas representadas na Figura 1 (variável MF) com os das curvas da Figura 2 (variável MS), houve menor variação na variável MS, o que, de certa forma, pode ser esperado, pela eliminação da variação que o teor de água pode causar sobre a determinação da MF.

A variável dano, obtida por avaliação visual, apresentou também curvas que ajustaram os valores atribuídos, com falta de ajuste não-significativo e valores de $\mathrm{I}_{50}$ também semelhantes aos obtidos nas outras variáveis (Figura 3). Embora os coeficientes de determinação tenham sido elevados, como nas demais variáveis, os coeficientes de variação das curvas de clomazone aplicado isoladamente e clomazone $+70 \mathrm{nM}$ de metribuzin foram bastante elevados (Figura 3B), sendo relacionado ao tipo de sintoma produzido pelo clomazone, em que o "branqueamento" provocado pode dificultar a atribuição mais precisa de um percentual de dano (Devine et al., 1993).

Analisando em conjunto os resultados das curvas de resposta às doses ajustadas para as três variáveis, verificou-se que em todas elas a associação dos herbicidas sempre reduziu a dose necessária para provocar 50\% do efeito avaliado. Em trabalho com associação de mecoprop-P e tribenuron-methyl sobre Veronica persica foi também observada redução dos $I_{50}$ para a variável $\mathrm{MF}$, com o incremento da proporção de tribenuron-methyl na associação (Streibig et al., 1998).

O isobolograma formado para a variável $\mathrm{MF}$, com a isobole de aditividade, traçada entre os $\mathrm{I}_{50}$ de metribuzin e clomazone aplicados isoladamente, com as faixas e intervalos de confiança para os $\mathrm{I}_{50}$ obtidos, estão na Figura 4. Os $I_{50}$ das quatro representações de associação entre os dois herbicidas estão abaixo da isobole de aditividade, estando na região considerada de interação sinérgica. No entanto, considerando-se as faixas de confiança $(\mathrm{FC})$ entre a isobole de aditividade e os IC dos $\mathrm{I}_{50}$ das associações, representados pelas barras verticais e horizontais, as associações de clomazone com metribuzin $140 \mathrm{nM}$ é que apresentam maior probabilidade de sinergismo, situando-se junto à FC de 64\%. Nessas associações houve em torno de $64 \%$ de probabilidade de atingir interação sinérgica. Portanto, a representação mencionada refletiu a $\mathrm{I}_{50}$ estimada pela curva de resposta às doses de clomazone (seis doses) em associação com 140 nM de metribuzin.

Para as demais associações, as probabilidades de sinergismo foram menores e mais variáveis (Figura 4), mas sempre se posicionando entre a região de sinergismo e a de

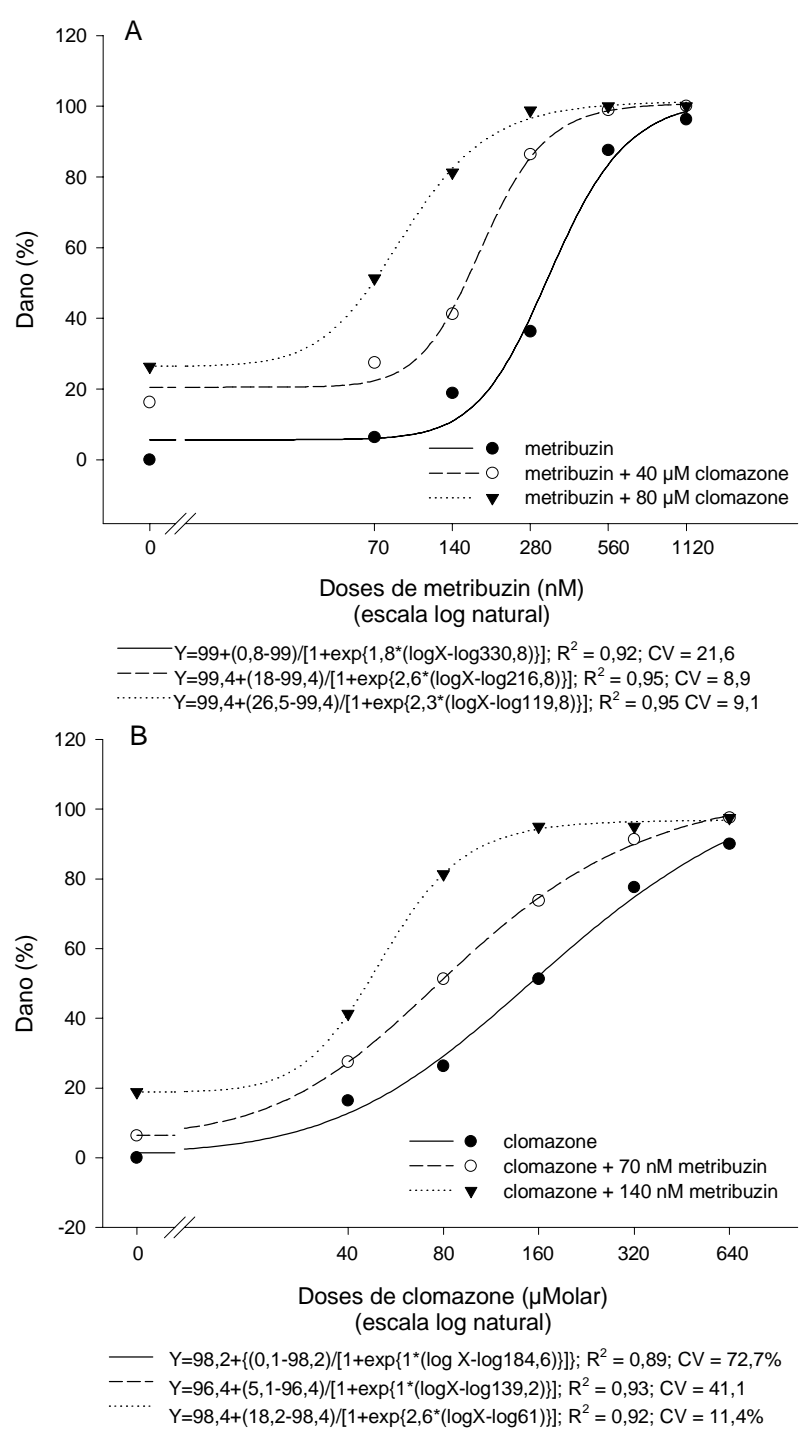

Figura 3 - Curvas de resposta do dano em girassol às doses dos herbicidas metribuzin (A) e clomazone (B), aplicados na solução nutritiva, isolados e em associação, quando as plantas apresentavam o segundo par de folhas completamente expandidas, avaliados aos cinco dias após a aplicação dos tratamentos. UFRGS, Porto Alegre, RS, 2001. 
aditividade. As associações de metribuzin e clomazone 40 PM e metribuzin e clomazone 80 PM situaram-se entre a FC de $64 \%$ e a isobole de aditividade, em que metribuzin e clomazone $40 \mu \mathrm{M}$ foi mais variável em seu IC, indo desde a FC de $64 \%$ até bem próximo da isobole de aditividade. Metribuzin e clomazone 80 PM, com IC menor, situou-se na região central entre a FC de $64 \%$ e a isobole de aditividade. Por fim, a representação das associações de clomazone e metribuzin $70 \mathrm{nM}$ situou-se entre a $\mathrm{FC}$ de $64 \%$ e a isobole de aditividade, porém com IC bastante amplo, variando desde a região entre $81 \%$ até além da isobole de aditividade, o que a torna menos informativa.

O isobolograma da variável MS indica uma região menos ampla em torno da isobole de aditividade, delimitada pela representação da FC (Figura 5). Isso refletiu a menor variação com que foram estimados os $\mathrm{I}_{50}$ para as curvas de resposta às doses de metribuzin e clomazone isolados para a variável MS. Por outro lado, como os $\mathrm{I}_{50}$ estimados para as duas variáveis foram equivalentes, as representações das associações posicionaram-se de forma semelhante diante da isobole de aditividade, mas também com menor variação em cada representação das associações na variável MS; em conseqüência, aumentou a probabilidade de sinergismo com a análise desta variável.

Novamente, as associações de clomazone e metribuzin $140 \mathrm{nM}$ destacaram-se, com probabilidade em torno de 0,81 (Figura 5). A variação expressa por seu IC permitiu prever a probabilidade de 81 a 95\% de obtenção de sinergismo nestas associações. As associações de metribuzin e clomazone 80 PM situaram-se entre 64 e $81 \%$, e as de metribuzin e clomazone 40 PM, entre menos de 64 até 90\%. Como na variável anterior, a representação das associações de clomazone e metribuzin $70 \mathrm{nM}$ foi a menos informativa, situando-se sobre a FC de $64 \%$, mas variando desde a isobole de aditividade até além da $\mathrm{FC}$ de $95 \%$.

O isobolograma que representa os resultados da variável dano foi o menos informativo (Figura 6). As representações das associações ficaram situadas mais próximas da isobole de aditividade, além de as FC e IC apresentaremse bem mais amplos. Provavelmente, a maior variabilidade encontrada nesta determinação (coeficiente de variação elevado) tenha gerado intervalos de confiança maiores em todos $\mathrm{I}_{50}$, o que provocou FC e IC mais extensos, inclusive com valores negativos, o que prejudica a análise da interação pelos resultados desta variável (Figura 6). No entanto, quando a curva de resposta às doses tem inclinação menos

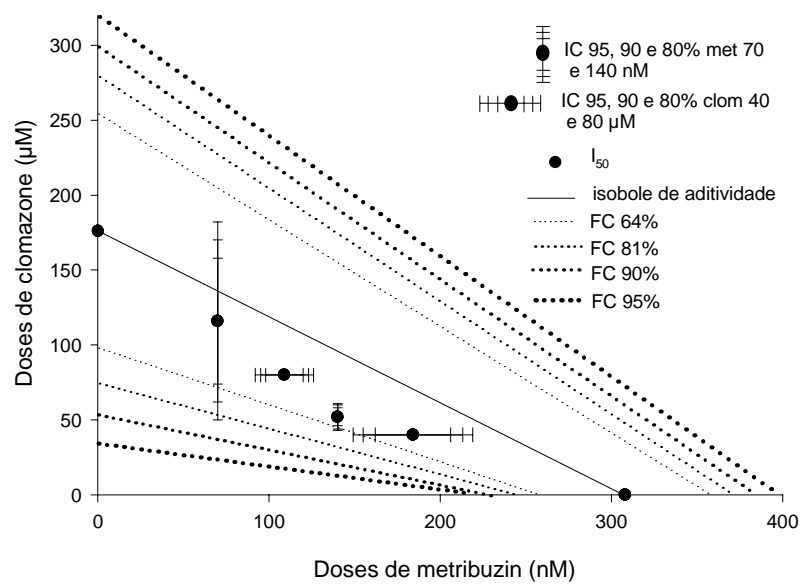

Figura 4 - Isobolograma da variável massa fresca de girassol com os valores da dose necessária para obter $50 \%$ de resposta $\left(\mathrm{I}_{50}\right)$, obtidos de curvas de resposta às doses de metribuzin (met) e clomazone (clom), aplicados isolados e em associação, com as faixas de confiança (FC) e intervalos de confiança (IC) correspondentes. UFRGS, Porto Alegre-RS, 2001.

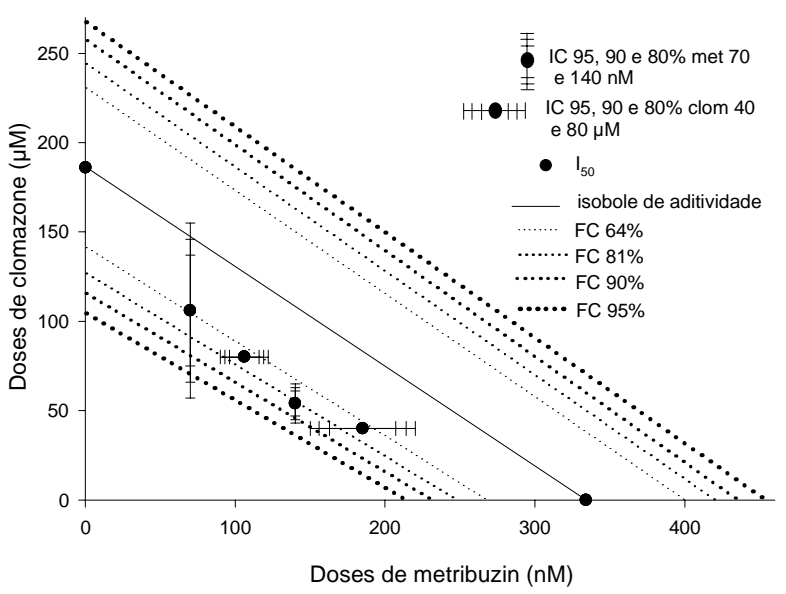

Figura 5 - Isobolograma da variável massa seca de girassol com os valores da dose necessária para obter $50 \%$ de resposta $\left(\mathrm{I}_{50}\right)$, obtidos de curvas de resposta às doses de metribuzin (met) e clomazone (clom), aplicados isolados e em associação, com as faixas de confiança (FC) e intervalos de confiança (IC) correspondentes. UFRGS, Porto Alegre-RS, 2001. 
acentuada, torna-se mais difícil estimar um limite inferior confiável para a curva logística (Streibig et al., 1999). De fato, quando se observam as curvas da variável dano, suas inclinações são as mais suaves, em comparação às variáveis MF e MS.

Trabalhos que empregam a mesma metodologia estão disponíveis na área da farmacologia, sendo verificadas interações de aditividade - supra-aditividade e infra-aditividade correspondentes a sinergismo e antagonismo, respectivamente. Esses trabalhos empregam com freqüência análises como a que foi aqui realizada, em que a posição relativa da representação das associações com a isobole de aditividade é tomada como indicativo da interação que está ocorrendo (Carter Jr., 1995; Gessner, 1995; Ramakrishnan \& Jusko, 2001). Em trabalho com benzoquinonas tóxicas, como sorgoleone, de origem natural e sintética, foi empregada metodologia similar, tentando demonstrar o mecanismo de ação do sorgoleone, como inibidor do FS II, pela comprovação de ausência de interação quando em associação com bentazon, herbicida inibidor do FS II (Streibig et al., 1999).

Outra forma referida de analisar a interação de agentes químicos é por meio do princípio de aditividade de Loewe, expresso pelo índice de combinação das associações de herbicidas $\left(\mathrm{IC}_{\text {omb }}\right)$ (Ramakrishnan \& Jusko, 2001). Na

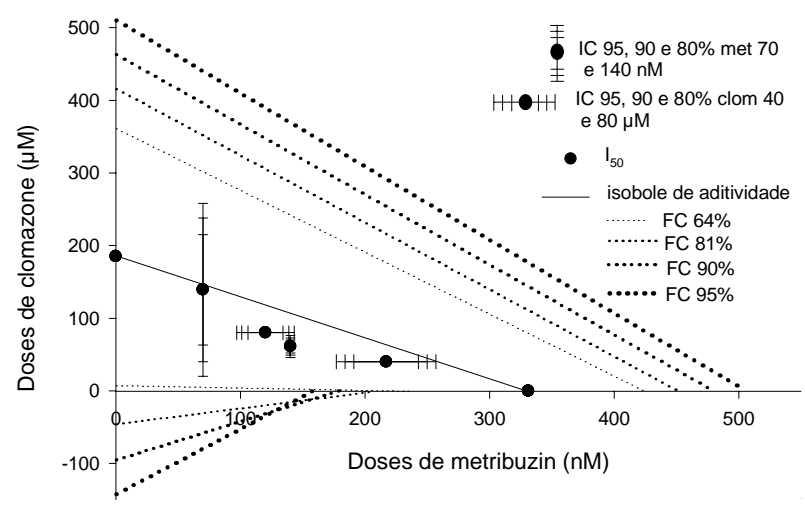

Figura 6 - Isobolograma da variável dano em girassol com os valores da dose necessária para obter $50 \%$ de resposta $\left(\mathrm{I}_{50}\right)$, obtidos de curvas de resposta às doses de metribuzin (met) e clomazone (clom), aplicados isolados e em associação, com as faixas de confiança (FC) e intervalos de confiança (IC) correspondentes. UFRGS, Porto Alegre-RS, 2001.
Tabela 1 estão os $\mathrm{IC}_{\text {omb }}$ para os quatro $\mathrm{I}_{50}$ resultantes das associações testadas. Nas três variáveis determinadas e em todas as associações, o índice calculado foi sempre inferior a um, indicando sinergismo na associação de metribuzin e clomazone por esse método.

Apesar de os isobologramas não mostrarem a existência de sinergismo em todas as associações com alto nível de probabilidade, também não houve indicativo de antagonismo, o que não pode ser descartado a priori, quando do estudo de qualquer tipo de associação (Green, 1989). Por outro lado, existem elementos que apontam para a existência de sinergismo, embora algumas associações, principalmente nas variáveis $\mathrm{MF}$ e dano, não se distingam da aditividade. De qualquer forma, fica saliente a necessidade de desenvolver mais aprimoradamente a metodologia de comparação entre os resultados da associação e a linha de aditividade teórica traçada entre os $\mathrm{I}_{50}$ dos produtos isolados. Além disso, o maior domínio dos fatores que provocam variabilidade experimental certamente contribuirá para a melhor comprovação do tipo de interação presente.

Com base nos resultados obtidos neste trabalho, é possivel afirmar que a variável massa seca permitiu comprovar, em algumas associações, a existência de sinergismo na associação dos herbicidas metribuzin e clomazone. A comprovação da existência de sinergismo pelo método de isobologramas depende da variável utilizada e da dose empregada na associação dos herbicidas.

Tabela 1 - Índice de combinação ( $\mathrm{IC}_{\mathrm{omb}}$ ) das associações de metribuzin e clomazone, calculado a partir das doses necessárias para obter $50 \%$ de resposta $\left(\mathrm{I}_{50}\right)$, obtidas a partir das curvas de resposta às doses destes herbicidas. UFRGS, Porto Alegre-RS, 2001

\begin{tabular}{|c|c|c|c|}
\hline \multirow{2}{*}{ Associação } & \multicolumn{3}{|c|}{$\mathrm{IC}_{\mathrm{omb}} *$} \\
\hline & $\mathrm{MF}^{1 /}$ & $\mathrm{MS}^{1 /}$ & Dano \\
\hline Metribuzin $+40 \mu \mathrm{M}$ clomazone & 0,82 & 0,77 & 0,87 \\
\hline Metribuzin $+80 \mu \mathrm{M}$ clomazone & 0,81 & 0,75 & 0,80 \\
\hline Clomazone + $70 \mathrm{nM}$ metribuzin & 0,89 & 0,78 & 0,97 \\
\hline Clomazone + $140 \mathrm{nM}$ metribuzin & 0,75 & 0,72 & 0,75 \\
\hline
\end{tabular}

* $\mathrm{IC}_{\text {omb }}=\mathrm{I}_{50 \mathrm{ab} 1} / \mathrm{I}_{50 \mathrm{a}}+\mathrm{I}_{50 \mathrm{ab} 2} / \mathrm{I}_{50 \mathrm{~b}}$, em que $\mathrm{I}_{50 \mathrm{ab} 1}$ e $\mathrm{I}_{50 \mathrm{ab} 2}$ são as doses $\mathrm{I}_{50}$ dos produtos em associação e $I_{50 a}$ e $I_{50 b}$, as doses $I_{50}$ dos produtos aplicados isoladamente.

1/ MF = massa fresca; MS = massa seca. 


\section{LITERATURA CITADA}

BLACKSHAW, R. E. et al. Response of triallate-resistant wild oat (Avena fatua) to alternative herbicides. Weed Technol., v. 10, n. 3, p. 258-262, 1996.

CAMPBELL, T. A.; GENTNER, W. A.; DANIELSON, L. L. Evaluation of herbicide interactions using linear regression moldeling. Weed Sci., v. 29, n. 4, p. 378-381, 1981.

CARTER JUNIOR, W. H. Relating isobolograms to response surfaces. Toxicology, v. 105, n. 12, p. 181-188, 1995.

CHISM, W. J.; BIRCH, J. B.; BINGHAM, S. W. Nonlinear regressions for analyzing growth stage and quinclorac interactions. Weed Technol., v. 6, n. 4, p. 898-903, 1992.

DEVINE, M.; DUKE, S. O.; FEDTKE, C. Physiology of herbicide action. Englewood Cliffs: PTR Prentice Hall, 1993. $441 \mathrm{p}$.

DRURY, R. E. Physiological interaction, its mathematical expression. Weed Sci., v. 28, n. 5, p. 573-579, 1980.

FRANS, R.; CROWLEY, H. Experimental design and techniques for measuring and analyzing plant responses to weed control practices. In: SOUTHERN WEED SCIENCE SOCIETY. Research methods in weed science. 3.ed. 1986. p. 29-45.

GESSNER, P. K. Isobolographic analysis of interactions: an update on applications and utility. Toxicology, v. 105, n. 12, 1995.

GOWING, D. P. Comments on tests of herbicide mixtures. Weeds, v. 8, n. 3, p. 379-391, 1960.

GREEN, J. Herbicide antagonism at the whole plant level. Weed Technol., v. 3, n. 2, p. 217-226, 1989.

GREEN, J. M.; BAILEY, S. P. Herbicide interactions with herbicides and other agricultural chemicals. In: McWHORTER, C. G.; GEBHARDT, M. R. Methods of applying herbicides. Champaign: WSSA, 1987. p. 37-61.

GREEN, J. M.; STREIBIG, J. C. Herbicide mixtures. In: STREIBIG, J. C.; KUDSK, P. Herbicide bioassays. Florida: CRC Press, 1993. p. 117-135.

GRESSEL, J. Synergizing herbicides. Rev. Weed Sci., v. 5. p. 49-82, 1990.

HATZIOS, K. K.; PENNER, D. Interactions of herbicides with other agrochemicals in higher plants. Rev. Weed Sci., v. 1. p. 1-63, 1985.
HEWITT, E. J. Sand an water culture methods used in the study of plant nutrition. 2.ed. East Maling:

Commonwealth Agricultural Bureau, 1966. 547 p.

JOHNSON, D. H.; YOUNG, B. G. Influence of temperature and relative humidity on the foliar activity of mesotrione. Weed Sci., v. 50, n. 2, p. 157-161, 2002.

MORSE, P. M. Some comments on the assessment of joint action in herbicide mixtures. Weed Sci., v. 26, n. 1, p. 58-71, 1978.

MOTULSKY, H. J.; RANSNAS, L. A. Fitting curves to data using nonlinear regression: a practical and nonmathematical review. FASEB J., v. 1, n. 5, p. 365-374, 1987.

RAMAKRISHNAN, R.; JUSKO, W. J. Interactions of aspirin and salicylic acid with prednisolone for inhibition of lymphocyte proliferation. Inter. Immunophar., v. 1, n. 12, 2001.

RIBOLDI, J. Cadernos de matemática e estatística: análise de variância. Porto Alegre: Universidade Federal do Rio Grande do Sul, 1995. 105 p. (Série B, 27)

SAS - INSTITUTE. Statistical Analysis System. User's guide. Version 6. 4.ed. Cary: 1989. 846 p.

SEEFELDT, S. S.; JENSEN, J. E.; FUERST, E. P. Loglogistic analysis of herbicide dose-response relationships. Weed Technol., v. 9, n. 2, p. 218-225, 1995.

SOUZA, A. P. et al. Uso da equação logística no estudo de dose-resposta de glyphosate e imazapyr por meio de bioensaios. Planta Daninha, v. 18, n. 1, p. 17-28, 2000

STREIBIG, J. C. Joint action of root-absorbed mixtures of auxin herbicides in Sinapis alba L. and barley (Hordeum vulgare L.). Weed Res., v. 27, n. 3, p. 337-347, 1987.

STREIBIG, J. C. et al. Joint action of natural and synthetic photosystem II inhibitors. Pest. Sci., v. 55, n. 2, p. 137-146, 1999.

STREIBIG, J. C.; KUDSK, P.; JENSEN, J. E. A general joint action model for herbicide mixtures. Pest. Sci., v. 53, n. 1, p. 21-28, 1998.

TAMMES, P. M. L. Isoboles, a graphic representation of synergism in pesticides. Nether. J. Plant Pathol., v. 70, n. 2, p. 73-80, 1964.

WRUBEL, R. P.; GRESSEL, J. Are herbicide mixtures useful for delaying the rapid evolution of resistance? a case study. Weed Technol., v. 8, n. 3, p. 635-648, 1994. 